\title{
Predicting the Culprit Lesion in Acute Inferior ST-Elevation Myocardial Infarction Based on Wellens' Criteria and Tierala's Algorithm
}

\author{
Kaveh Hosseini ${ }^{1}$; Ali Bozorgi ${ }^{2}$; Shahrokh Karbalayi ${ }^{1, *}$ \\ ${ }^{1}$ Cardiology Departement, Sina Hospital, Tehran University of Medical Sciences, Tehran, IR Iran

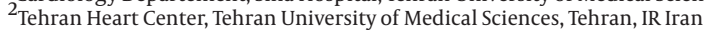 \\ ${ }^{*}$ Corresponding author: Shahrokh Karbalayi, Cardiology Department, Sina Hospital, Hassan'abad square, Imam Khomeini Street, Tehran University of Medical Sciences, Tehran, IR \\ Iran. Tel: +98-9122014164, Fax:+98-2166348555, E-mail: saleh6948@yahoo.com
}

Received: October 23, 2013; Revised: December 3, 2013; Accepted: December 7, 2013

\begin{abstract}
Background: Defining the infarct related artery in acute myocardial infarction helps in better and faster management of patients. Therapeutic choices may differ according to the culprit lesion.

Objectives: This study aimed to evaluate multiple electrocardiography (ECG) criteria and one algorithm in defining the culprit artery in single vessel inferior ST elevation myocardial infarction(I-STEMI). A new criterion based on posterior leads was also proposed.

Materials and Methods: In this retrospective study from June 2007 to July 2012, ECG and angiography films of patients with acute inferior STEMI were reviewed. From a total of 138 studied patients, 25 had 3-vessel disease and 37 had two occluded arteries. Remaining 76 patients were diagnosed with single vessel acute I-STEMI, 56 had right coronary artery (RCA) occlusion [22 (29.3\%) proximal RCA, 24 (32\%) middle RCA, and 10 (13.3\%) distal occlusion of RCA], 19 had left circumflex artery (LCX) lesion and one had middle left anterior descending (LAD) artery occlusion.

Results: Prediction of the RCA as culprit lesion using Tierala's algorithm was $86 \%$ sensitive and $50 \%$ specific. Prediction of LCx occlusion based on ST-elevation $\geq 1 \mathrm{~mm}$ in V6 was $87 \%$ specific $(\mathrm{P}=0.005)$. Sum of ST elevation in leads V5 and V6 more than $2.5 \mathrm{~mm}$, was a good marker of LCx prediction $(P=0.044)$. ST-elevation in V4R was $48 \%$ sensitive and $89 \%$ specific for RCA prediction $(P=0.004)$. Wellens' criterion was $82 \%$ sensitive and $47 \%$ specific for proximal RCA prediction $(\mathrm{P}=0.002)$. Our new criterion "Sum of ST elevation in posterior leads $(\mathrm{V7}-\mathrm{V} 8$ - V9 $)<3 \mathrm{~mm}$ " was $82 \%$ sensitive and $50 \%$ specific for RCA prediction $(\mathrm{P}=0.017)$. We also revised Tierala's algorithm by adding the presence of ST-elevation in V3 and V4 to the first step (when STe II $\geq$ III), which increased the specificity and PPV of LCx prediction (86\% vs. $84 \%$ and $53 \%$ vs. $50 \%$ ).

Conclusions:Although several criteria and algorithms were previously suggested, they could not reliably determine the site of occlusion. Right and posterior leads may be needed in order to increase the accuracy of prediction.
\end{abstract}

Keywords: Coronary Angiography; Electrocardiography; Myocardial Infarction

\section{Background}

Electrocardiography (ECG) as the first step in managing the patients with acute chest pain may help defining the culprit lesion before performing invasive steps. However, exact determination of ECG-culprit lesion relationship is confounded by multi-vessel involvement; thus, we only enrolled patients with single vessel inferior ST elevation myocardial infarction (I-STEMI). Stenosis of proximal right coronary artery (PRCA) may lead to devastating outcomes because of right ventricular (RV) failure, shock, and/or hypotension (1). Distal lesions of left circumflex (LCX) compared with proximal lesions of RCA or LCx is not associated with poor prognosis $(2,3)$. To differentiate between RCA and LCX, several algorithms and criteria were previously proposed, but their sensitivity and specificity were limited (4). In this study, the main is- sue was focused on the fact that whether 12-lead ECG and right precordial leads could identify the infarct related artery. Adding more leads, posterior leads may also help in emergent decision-making. The clinical point of view about categorizing high-risk patients with right ventricular involvement is of great value and importance. In 1984, right precordial lead V4R was proposed as another marker to predict the right ventricular involvement. As we routinely record right precordial leads in emergency wards in training hospitals, it was worthy to evaluate this criterion in our sample size. In the recent studies, in addition to several criteria, some algorithms were also proposed; for example, in 2004 and 2009 two interesting algorithms were designed and published by two different researchers. categorize patients in high and low risk groups and choose individualized treatments.

Copyright (C) 2014, Kowsar Corp. This is an open-access article distributed under the terms of the Creative Commons Attribution License, which permits unrestricted use, distribution, and reproduction in any medium, provided the original work is properly cited. 


\section{Objectives}

In this study, we decided to evaluate the sensitivity and specificity of previously-suggested criteria and one algorithm. We hope in the future meta-analysis of past studies will reveal the most accurate and practical criteria and/or algorithm.

\section{Materials and Methods}

In this retrospective study, we enrolled patients admitted in the Emergency Department (ED) of Tehran Heart Center (THC) between 23 June 2007 and 2 July 2012 because of acute I-STEMI. They underwent percutaneous coronary intervention during their hospitalization. Basis of the diagnosis of I-STEMI was acute typical chest pain with increased cardiac markers and ST elevation of $\geq 0.5$ $\mathrm{mm}$ in two of the three inferior leads. ST changes were measured at $80 \mathrm{~ms}$ from J points, and the TP segment was the isoelectric line of the ECG. ST deviations $<0.5$ $\mathrm{mm}$ in frontal leads and $<1 \mathrm{~mm}$ in precordial leads were regarded as isoelectric. Significant stenosis was considered as $>70 \%$ luminal narrowing. The mean ST deviations of three consecutive beats were reported as the final ST deviations. In almost 10\% of ECG reports there was $>0.5$ $\mathrm{mm}$ difference, and they were re-evaluated in the presence of two cardiologists at the same time. There was no disagreement in angiographic reports. We excluded multi-vessel occlusion, left bundle branch block, left ventricular hypertrophy, prior history of coronary artery bypass graft, and prior myocardial infarction. Two cases of acute I-STEMI with "wrap-around" left anterior descending (LAD) occlusion at distal parts with concomitant RCA involvement were also excluded. There was one case of single vessel apical myocardial infarction (MI) due to mid portion occlusion of LAD which was included in the analysis. Posterior leads were recorded in the THC emer- gency ward; so we analyzed the association between ST deviations in these three leads with the site of occlusion. In our analysis 76 patients were included, 75 of which had significant narrowing limited to RCA or LCx and one had occlusion of the mid portion of LAD.

Patients' on-admission-ECGs were interpreted by two skilled cardiologists. They were blinded to the angiographic reports. The same strategy was also performed for the angiographic data. ECGs were recorded within six hours of the symptoms onset. The maximum time between the symptoms onset and coronary angiography was less than 6 days during the hospitalization period. Tierala's algorithm was analyzed in terms of its accuracy in defining the site of occlusion in acute I-STEMI. Patients with Wellens et al. (5) inclusion criteria were separately analyzed.

\subsection{Statistical Analysis}

We reported continuous variables in terms of means \pm standard deviation and for discrete variables, frequencies and percentages. We compared means of continuous variables with independent samples T-test. Correlations of discrete variables were measured by chi-square test of independence. P value $<0.05$ was considered statistically significant. Data analysis was performed using the SPSS version 13.0 statistical package (Chicago, Illinois). For all criteria, sensitivity, specificity, and predictive values were calculated.

\section{Results}

Demographic and clinical features of the patients are shown in Table 1. Culprit arteries based on angiographic repots are illustrated in Table 2 . All 76 patients were analyzed according to the algorithms of Tierala et al. (6) and prespecified criteria (Tables 3 and 4).

\begin{tabular}{|c|c|c|c|}
\hline Characteristics & Group 1 (RCA), n = 56 & Group $2(\mathrm{LCx}), \mathrm{n}=19$ & Group 3 (LAD), $\mathbf{n}=1$ \\
\hline Age, $y$ & $56 \pm 10$ & $57 \pm 12$ & 55 \\
\hline Gender, female & $9(16)$ & $6(32)$ & $0(0)$ \\
\hline Hypertension & $11(19.6)$ & $6(31.6)$ & $0(0)$ \\
\hline Diabetes mellitus & $12(21.4)$ & $5(26.3)$ & $1(100)$ \\
\hline Dyslipidemia & $20(35.7)$ & $8(42.1)$ & $1(100)$ \\
\hline Smoking & $30(53.7)$ & $10(52.6)$ & $1(100)$ \\
\hline$<10$ pack $/ y$ & $18(32.1)$ & $6(31.5)$ & $0(0)$ \\
\hline $10-20$ pack/y & $8(14.2)$ & $3(15.7)$ & $0(0)$ \\
\hline$>20$ pack/y & $4(7.14)$ & $1(5.2)$ & $1(100)$ \\
\hline $\begin{array}{l}\text { Left ventricular ejection fraction at } \\
\text { the time of admission, } \%\end{array}$ & $49.5 \pm 6$ & $47 \pm 6$ & 44 \\
\hline
\end{tabular}

a Abbreviations: LAD, left anterior descending artery; LCx, left circumflex artery; RCA, right coronary artery.

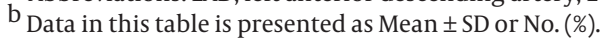


Hosseini Ket al.

\begin{tabular}{ll}
\hline Table 2. Infarct-Related Artery Based on Angiography Reports in I-STEMI & a $(\mathrm{n}=76)$ \\
\hline Culprit artery & Distribution, No. $\mathbf{0})$ \\
\hline Right coronary artery & $56(74.7)$ \\
\hline Proximal & $22(29.3)$ \\
\hline Middle & $24(32)$ \\
\hline Distal & $10(13.3)$ \\
\hline Left circumflex artery & $19(25.3)$ \\
\hline Proximal & $7(9.3)$ \\
Nonproximal & $11(14.6)$ \\
\hline Obtuse marginal branch & $1(1.3)$ \\
\hline Left anterior descending artery & $1(1.3)$ \\
\hline Mid portion & $1(1.3)$ \\
\hline
\end{tabular}

a Abbreviation: I-STEMI, inferior ST-elevation myocardial infarction.

Table 3. Correlations of ST-segment Deviation and the Culprit Artery in I-STEMI, in Prespecified Criteria a

\begin{tabular}{|c|c|c|c|c|c|c|c|}
\hline Criteria & $\begin{array}{l}\text { LCx as a Culprit Ar- } \\
\text { tery, } n=19 \text {, No. }(\%)\end{array}$ & $\begin{array}{l}\text { RCA as a Culprit Ar- } \\
\text { tery, } n=56 \text {, No. }(\%)\end{array}$ & Sensitivity & Specificity & PPV & NPV & PValue \\
\hline STe in III $\geq$ II & $8(42 \%)$ & $54(96 \%)$ & $96 \%$ for RCA & $45 \%$ for RCA & $83 \%$ for RCA & $82 \%$ for RCA & 0.000 \\
\hline STd in I $\geq 0.5 \mathrm{~mm}$ & $7(36 \%)$ & $38(67 \%)$ & $68 \%$ for RCA & $65 \%$ for RCA & $84 \%$ for RCA & $42 \%$ for RCA & 0.017 \\
\hline STe V6 $\geq 1$ & $8(42 \%)$ & $7(12.5 \%)$ & $42 \%$ for $\mathrm{LCX}$ & $87 \%$ for $\mathrm{LCX}$ & $53 \%$ for LCx & $82 \%$ for $\mathrm{LCx}$ & 0.005 \\
\hline$S T e$ in $V 4 R \geq 1 \mathrm{~mm}$ & $2(10 \%)$ & $27(48 \%)$ & $48 \%$ for RCA & $89 \%$ for RCA & $93 \%$ for RCA & $40 \%$ for RCA & 0.004 \\
\hline
\end{tabular}

a Abbreviations: e, elevation; d, depression; NPV, negative predictive value; $\mathrm{LCx}$, left circumflex artery; PPV, positive predictive value; RCA, right coronary artery.

Table 4. Sensitivity, Specificity and Predictive Values of Each Step of Algorithms ${ }^{a}(n=76)$

\begin{tabular}{llllll}
\hline Tierala's Algorithm Steps & Culprit Artery & Sensitivity, $\%$ & Specificity, $\%$ & PPV, $\%$ & NPV, $\%$ \\
\hline Step 1:ST elevation II $\geq$ III & LCX & 42 & 91 & 62 & 83 \\
Step 2: part A and part B & RCA & 48 & 36 & 78 & 13 \\
Step 3: ST depression aVR $\geq$ aVL & LCX & 25 & 85 & 20 & 88 \\
\hline
\end{tabular}

a Abbreviations: LCx, left circumflex artery; NPV, negative predictive value; PPV, positive predictive value; RCA, right coronary artery.

\subsection{Tierala's Algorithm Analysis}

Tierala's Algorithm is presented in Figure 1 and Table 4. In our study, 13 patients had STeII $\geq$ III and according to the first step of this algorithm, eight of them were truly defined as LCx. Sixty-three patients had ST elevation III > II, analyzed in the second step. We categorized the second step in two parts: A and B. In part A and B of the second step, Tierala algorithm defined seven and 25 patients as RCA occlusion, respectively, 25 of which had been primarily predicted as RCA. We still need an additional step to define all of the culprit arteries. The last step compared ST depression in aVR and aVL. This step could truly define one LCX and 23 RCAs. Based on the Tieral's algorithm, our patient with LAD lesion and resulting apical MI had been predicted to have LCx occlusion. Values of three steps together are shown in Table 5. Reported sensitivity and specificity for RCA as the occluded vessel in Tierala et al. (6) algorithm were $96 \%$ and $56 \%$, respectively. Positive predictive value (PPV) was $92 \%$ and negative predictive value (NPV) was 75\% in their study.

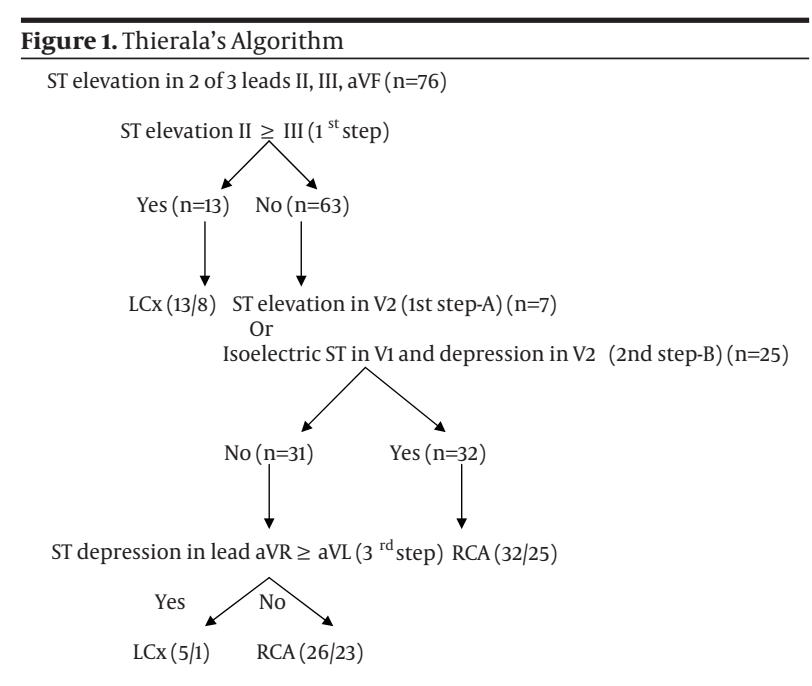

ST elevation in two of three leads II, III, aVF $(n=76)$ 
Table 5. Sensitivity, Specificity and Predictive Values for Tierala's Algorithms a $(n=76)$

\begin{tabular}{lllll}
\hline ECG algorithm & Sensitivity, $\%$ & Specificity, $\%$ & PPV, $\%$ & NPV, $\%$ \\
\hline Tierala & & & & \\
RCA & 86 & 50 & 83 & 56 \\
LCX & 47 & 84 & 50 & 83 \\
\hline
\end{tabular}

a Abbreviations: ECG, electrocardiogram; LCx, left circumflex artery; NPV, negative predictive value; PPV, positive predictive value.

Our case with apical MI and LAD lesion had ST elevation in leads II and III (STe II > III) and V3 to V6. Right precordial leads were not deviated in this case. It was predicted as LCX in Tierala's algorithms. We added the presence of ST-elevation in V3 and V4 to the first step (when STe II $\geq$ III), which increased the specificity and PPV of LCx prediction compared to Tierala's main algorithm ( $86 \%$ vs. $84 \%$ and $53 \%$ vs. $50 \%$ ).

Evaluation of right precordial leads based on Wellens criteria: ST elevation $\geq 1 \mathrm{~mm}$ in V4R or isoelectric V4R combined with positive T wave was considered as RCA occlusion. In addition, ST depression $\geq 1 \mathrm{~mm}$ with a negative T-wave was LCx based on Wellens criteria. Therefore, 52 patients were analyzed based on Wellens' criteria. Occluded artery in these patients was RCA or LCX. Wellens criteria were $97 \%$ sensitive and $36 \%$ specific for RCA prediction. Positive and negative predictive values were $85 \%$ and $80 \%$, respectively. Wellens' criteria significantly predicted the PRCA as the culprit lesion $(\mathrm{P}=0.002)$. In this case, sensitivity and specificity were $82 \%$ and $47 \%$ respectively for PRCA prediction.

Our new criterion "Sum of ST elevation in posterior leads (V7-V8-V9) $<3$ mm" was $82 \%$ sensitive and 50\% specific for RCA prediction ( $\mathrm{P}=0.017)$. In our database, posterior leads were recorded in 68 patients. Sum of ST elevation $<3 \mathrm{~mm}$ was also significantly related to the site of occlusion within the vessels $(\mathrm{P}=0.0016)$. We categorized RCA involvement in three (proximal, middle, and distal) and LCx occlusion in two groups (proximal and nonproximal). ST elevation $<3 \mathrm{~mm}$ was seen in $85 \%$ (17/20) of proximal, $88 \%(21 / 24)$ of middle, and $100 \%$ (8/8) of distal RCAs.

\section{Discussion}

RCA supplies blood for RV and some parts of the left ventricle. In most of the people, RCA also gives off the posterior descending artery (PDA) (7). RCA supplies blood for the inferior wall and posterior parts of the septum. LCX supplies the postero-lateral wall and posterior part of the inferoposterior septum. In $13.3 \%$ of the population, LCX gives rise to PDA (7).

The proposed criteria are based on vectors of injury, as in the case of RCA, results in infarction of more inferior parts of the heart, compared with posterolateral parts. The vector of injury towards lead III causes more ST eleva- tion in lead III compared with lead II (8). The same insight is applied to LCx occlusion. Based on the literature review, in acute I-STEMI we can categorize all proposed criteria in four subdivisions: ST elevation in inferior leads; ST deviations in other leads based on the injury vector (5); reciprocal changes in I, aVL, V1 to V3 (9); and finally right precordial leads (5).

Recording right precordial leads are not routine in ED as the first on-admission ECG, so the reported sensitivity and specificity of those criteria based on them is limited. ST elevation in V4R is a reliable marker in cases of right ventricle (RV) involvement and proximal RCA occlusion (5, 7-10). Besides the presence of the elevation, the magnitude of deviation in the right precordial leads is an important determinant of prediction (5). ST-deviation in aVR is also considered as another marker for culprit artery prediction. According to this vector theory, other leads may also come to work to predict the site of occlusion. As an instance, one study from Iran in 2012 in Yazd Medical University, analyzed ST elevation in aVR as an indicator for left main artery obstruction which had sensitivity and specificity of $62.7 \%$ and $73.6 \%$, respectively (11).

In some cases of middle or distal occlusion of LAD and apical MI, the ECG pattern may be similar to occlusion of RCA or LCX. In these cases, we may have I-STEMI and concomitant ST elevation in anterolateral leads. To deal with this issue, we revised Tierala's algorithm by adding a new step as ST elevation in V3 and V4.

We can't compare Wellens' criteria with Tierala's, because of unequal sample sizes. However, with high-probability assessing and reporting right precordial leads are associated with good sensitivity in right ventricular involvement. Right precordial leads changes may disappear in early stages of STEMI (8). Therefore, we should seriously consider the time lapse between the onset of symptoms and ECG recording.

\subsection{Study limitations}

Obviously, inferior STEMI is not always limited to just one vessel occlusion and in one site; thus, this study is less helpful to determine the exact value of the examined criteria in patients with multi-vessel involvement. Small number of patients, especially patients with LCx occlusion is another limitation of these kinds of studies.

To deal with the beat to beat variability of ST segment deviation, we calculated the mean of three consecutive beats as the final ST deviation reports.

\section{Acknowledgments}

The authors thank Mehran Mahmoudian MD, for providing well-defined database for our analysis and Soheil Saadat MD, for epidemiologic consult. We thank Sherry Hughes Garner and Kimia Najafi for editing the manuscript. Our gratitude goes out as well to The Research Division and Database Registry Section of Tehran Heart Center. 


\section{Authors' Contribution}

First idea: Dr. karbalayi. Data gathering, statistical analysis, and paper writing: Kaveh Hosseini. Academic guidance: Dr. Bozorgi and Dr. Karbalayi. Angiography and ECG reporting: Dr. bozorgi and Dr. Karbalayi.

\section{Financial Disclosure}

There was no conflict of interest in this article.

\section{Funding/Support}

There was no financial support.

\section{References}

1. Braat SH, de Zwaan C, Brugada P, Coenegracht JM, Wellens HJ Right ventricular involvement with acute inferior wall myocardial infarction identifies high risk of developing atrioventricular nodal conduction disturbances. Am Heart J. 1984;107(6):1183-7.

2. Karha J, Murphy SA, Kirtane AJ, de Lemos JA, Aroesty JM, Cannon $\mathrm{CP}$, et al. Evaluation of the association of proximal coronary culprit artery lesion location with clinical outcomes in acute myocardial infarction. Am J Cardiol. 2003;92(8):913-8.

3. Bates ER, Califf RM, Stack RS, Aronson L, George BS, Candela RJ, et al. Thrombolysis and Angioplasty in Myocardial Infarction (TAMI-1) trial: influence of infarct location on arterial patency, left ventricular function and mortality. J Am Coll Cardiol. 1989;13(1):12-8.
4. Kosuge M, Kimura K, Ishikawa T, Hongo Y, Mochida Y, Sugiyama $\mathrm{M}$, et al. New electrocardiographic criteria for predicting the site of coronary artery occlusion in inferior wall acute myocardial infarction. Am J Cardiol. 1998;82(11):1318-22.

5. Wellens HJ. The value of the right precordial leads of the electrocardiogram. N Engl J Med.1999;340(5):381-3.

6. Tierala I, Nikus KC, Sclarovsky S, Syvanne M, Eskola M. Predicting the culprit artery in acute ST-elevation myocardial infarction and introducing a new algorithm to predict infarct-related artery in inferior ST-elevation myocardial infarction: correlation with coronary anatomy in the HAAMU Trial. J Electrocardiol. 2009;42(2):120-7.

7. Vales L, Kanei Y, Schweitzer P. Electrocardiographic predictors of culprit artery in acute inferior ST elevation myocardial infarction. J Electrocardiol. 2011;44(1):31-5.

8. Fiol M, Cygankiewicz I, Carrillo A, Bayes-Genis A, Santoyo O, Gomez A, et al. Value of electrocardiographic algorithm based on "ups and downs" of ST in assessment of a culprit artery in evolving inferior wall acute myocardial infarction. Am J Cardiol. 2004;94(6):709-14.

9. Hira RS, Wilson JM, Birnbaum Y. Introducing a new algorithm in inferior ST-segment elevation myocardial infarction to predict the culprit artery and distinguish proximal versus distal lesions. Coron Artery Dis. 2011;22(3):165-70.

10. Erhardt LR, Sjogren A, Wahlberg I. Single right-sided precordial lead in the diagnosis of right ventricular involvement in inferior myocardial infarction. Am Heart J. 1976;91(5):571-6.

11. Nough H, Jorat MV, Varasteravan HR, Ahmadieh MH, Tavakkolian N, Sheikhvatan M. The value of ST-segment elevation in lead aVR for predicting left main coronary artery lesion in patients suspected of acute coronary syndrome. Rom J Intern Med. 2012;50(2):159-64. 